Gynäkologe 2020 · 53:412-413

https://doi.org/10.1007/s00129-020-04611-5

Online publiziert: 19. Mai 2020

(c) Springer Medizin Verlag GmbH, ein Teil von Springer Nature 2020

\author{
Rolf Kreienberg' ${ }^{1}$ Andreas Schröer ${ }^{2} \cdot$ Alexander Strauss $^{3} \cdot$ Klaus Vetter $^{4} \cdot$ \\ Klaus Diedrich ${ }^{5}$. Stefanie Schumacher-Schmidt ${ }^{6}$ \\ 'Mainz, Deutschland \\ ${ }^{2}$ Zentrum für Pränataldiagnostik und Humangenetik, Berlin, Deutschland \\ ${ }^{3}$ Christian-Albrechts-Universität zu Kiel, Kiel, Deutschland \\ ${ }^{4}$ Berlin, Deutschland \\ ${ }^{5}$ Department of Gynecology and Obstetrics, Universitätsklinikum Schleswig-Holstein, Lübeck, \\ Deutschland \\ ${ }^{6}$ Fachzeitschriften Medizin, Springer Medizin Verlag GmbH, Heidelberg, Deutschland
}

\title{
Neue Rubrik in Der Gynäkologe: Handlungsalgorithmus
}

Liebe Leserinnen und Leser,

In den letzten Jahren haben sich im gesamten Gesundheitssystem viele Änderungen ergeben, die sich erheblich auf die Abläufe des medizinischen Handelns in Praxis und Klinik auswirken. Medizinische Qualität auf den Grundlagen einer evidenzbasierten wissenschaftlichen Medizin geht mit der rasanten Etablierung neuer, mitunter kostenaufwendiger Techniken, geänderten Abrechnungsverfahren und wachsendem Kostendruck einher.

Leitlinien fassen die aktuelle Studienlage zusammen und geben Empfehlungen bzw. öffnen Handlungskorridore für den ärztlichen Alltag vor. Diese werden regelmäßig aktualisiert. Sie sind jedoch mitunter umfangreich und daher manchmal wenig übersichtlich zu lesen. Die Arbeitsgemeinschaft der Wissenschaftlichen Medizinischen Fachgesellschaften (AWMF, www.awmf.de) und die Deutsche Gesellschaft für Gynäkologie und Geburtshilfe (DGGG, www. dggg.de) informieren dazu regelmäßig über ihre Newsletter. Die Herausgeber*innen präsentieren die Inhalte der Leitlinien in Der Gynäkologe in Form von CME(„continuing medical education“)Beiträgen oder ganzen Themenheften. Grundsätzlich ist es empfehlenswert, diese Leitlinien ausführlich $\mathrm{zu}$ studieren. Für die rasche Intervention und zur Diskussion in der Praxis oder am Krankenbett kommen allerdings auch kurzgefasste Handlungsempfehlungen den alltäglichen Bedürfnissen entgegen.

Um den Leser*innen von Der Gynäkologe einen schnellen Überblick zu einem wichtigen und praktisch relevanten Thema zu geben, wurde daher die Rubrik „Handlungsalgorithmus“ gegründet. Sie soll dazu dienen, Strategien graphisch nachvollziehbar zu präsentieren. Dabei kann es sich um Inhalte aus Leitlinien, aber auch um nicht formal abgesegnete Vorgehensweisen handeln.

\section{I) Schneller Überblick zu wichtigen und praktisch relevanten Themen}

Die Beiträge dieser Rubrik werden einmal im Quartal als Doppelseite im Heft präsentiert: Auf der linken Seite werden die Schritte und wichtige Informationen zum Algorithmus stichpunktartig beschrieben. Auf der rechten Seite ist der Algorithmus selbst graphisch dargestellt.

Die Vorschläge der Autor*innen folgen praktischen Überlegungen und können jederzeit diskutiert und aktualisiert werden. Sie sind als strategische Vorlage, nicht aber als rechtlich bindende Vorgaben $\mathrm{zu}$ verstehen.

Als Rubrikherausgeber fungieren Herr Prof. Dr. Rolf Kreienberg, Herr PD Dr. Andreas Schröer, Herr Prof. Dr. Alexander Strauss sowie Herr Prof. Dr. Klaus Vetter. Sie planen die Themen, laden die 


\section{Aktuelle Informationen}

Autor*innen ein und begutachten die Artikel vor der Publikation.

Wir wünschen Ihnen eine gewinnbringende Lektüre des ersten Handlungsalgorithmus zur „Extrauterinen Gravidität" von Frau Dr. Ramsauer in diesem Heft. Über Ihre konstruktiven Rückmeldungen freuen wir uns sehr.

Ihre<smiles>CCCCCCCCCCCCCCCCCCC</smiles>

Rolf Kreienberg

\section{Andreas felvöes}

Andreas Schröer

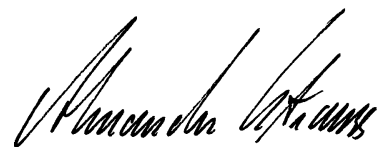

Alexander Strauss

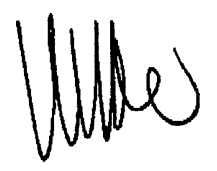

Klaus Vetter

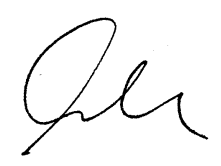

Klaus Diedrich

S6. Solumaler-Schmicht

Stefanie Schumacher-Schmidt

\section{Korrespondenzadresse}

\section{Dr. Stefanie Schumacher-Schmidt}

Fachzeitschriften Medizin, Springer Medizin

Verlag $\mathrm{GmbH}$

Tiergartenstr. 17, 69121 Heidelberg,

Deutschland

stefanie.schumacher@springer.com

Interessenkonflikt. R. Kreienberg, A. Schröer, A. Strauss, K. Vetter, K. Diedrich und S. SchumacherSchmidt geben an, dass kein Interessenkonflikt besteht.

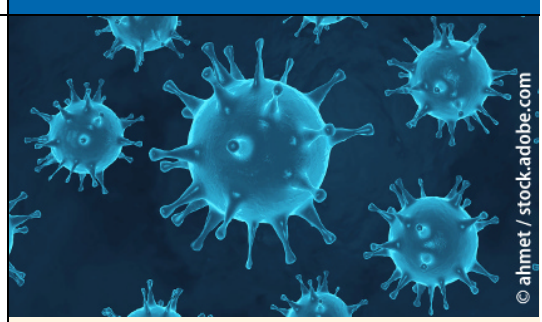

\section{Aktuelle Entwicklung zu COVID-19 bei Springer Nature und Springer Medizin}

Springer Nature und Springer Medizin unterstützen die globale $\mathbf{R e}$ aktion auf die COVID-19-Pandemie, indem ein schneller und direkter $\mathrm{Zu}$ gang zu den neuesten verfügbaren Forschungsergebnissen und Daten ermöglicht wird.

Auf der Homepage SpringerMedizin.de finden Sie ein immer aktuelles Dossier mit Beiträgen, Forschungsarbeiten und Ergebnissen zu SARS-CoV-2 sowie relevanten Links.

Darin z.B. auch die kürzlich publizierte

Empfehlung von DIVI, DGIIN, DGAI und DGP zur Intensivtherapie von Patienten mit COVID-19.

Springer Nature arbeitet mit globalen Organisationen zusammen, und verlinkt über SpringerNature.com/de auf eine eigene Landingpage mit einer Vielzahl an Information sowie freiem Zugriff auf die COVID-19-Contentplattformen von Nature Research, BioMed Central (BMC) und Springer

Das Dossier zu Coronavirus / Covid-19 von Springer Medizin finden Sie hier: www.springermedizin.de/covid-19

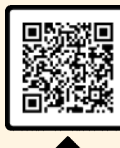

SCAN ME 